\title{
Aplicación de los diagramas de Karnaugh - veitch, en el diseño de circuitos neumáticos con señales blocantes.
}

Implementation of the karnaugh diagrams - veitch, in the design of pneumatic circuits with signs-blocking rules

Edgar Fabián Sánchez Carrión. ${ }^{1}$, Elvis Enrique Arguello. ${ }^{2}$, José Pérez Fiallos. ${ }^{3}$ \& Gonzalo Noboa Larrea. ${ }^{4}$

Recibido: 05-12-2017 / Revisado: 12-02-2018 Aceptado: 10-03-2018/ Publicado: 01-04-2018

\begin{abstract}
.
DOI: https://doi.org/10.33262/cienciadigital.v2i2.113

This article presents the development of the Karnaugh - Veitch diagrams, the application of the Morgan theorem of Boolean algebra and logical functions, AND, OR and NOT and their respective pneumatic equivalences, for the implementation in the design of pneumatic circuits with blocking signals.
\end{abstract}

Keywords: Karnaugh-Veitch Diagrams, Logic Functions, Pneumatic Circuit, Blocking Signals.

\section{Resumen.}

Este artículo presenta el desarrollo de los diagramas de Karnaugh-Veitch, la aplicación de los teorema de Morgan del algebra booleana y funciones lógicas, YES, NOT, AND y OR, con sus respectivas equivalencias neumáticas, para la implementación en el diseño de circuitos neumáticos con señales blocantes.

\footnotetext{
${ }_{1}$ Escuela Superior Politécnica de Chimborazo, Chimborazo, Ecuador, esanchez_c@espoch.edu.ec

${ }^{2}$ Escuela Superior Politécnica de Chimborazo, Chimborazo, Ecuador, earguello@espoch.edu.ec

${ }^{3}$ Escuela Superior Politécnica de Chimborazo, Chimborazo, Ecuador, jperez@espoch.edu.ec

${ }^{4}$ Escuela Superior Politécnica de Chimborazo, Chimborazo, Ecuador, procuraduría@ueb.edu.ec
} 
Palabras clave: Diagramas de Karnaugh-Veitch, Funciones Lógicas, Circuito Neumático, Señales Blocantes

\section{Introducción .}

El diagrama de Karnaugh-Veitch (K-V) se usa con amplitud para simplificar problemas intrincados de control, los cuales proporcionan una representación gráfica de las variables de control y ayudan a formar ecuaciones del álgebra booleana, estos diagramas pueden contener un gran número de variables de control en sus modos algebraicos y lógico binario. A partir del diagrama se pueden combinar en varias ecuaciones booleanas adecuadas, aplicando los teoremas de Morgan. La máxima ventaja del diagrama $\mathrm{K}-\mathrm{V}$ se apoya en su capacidad para abarcar cualquier número de variables en la forma matemática de su modo "SI" o "NO", a través de un bloque cuadrado que se divide en varios campos, en cada uno de los cuales se indica la presencia o la ausencia de una señal (variable).

Las funciones lógicas YES y NOT, tienen sus equivalentes en las válvulas neumáticas en sus estados normalmente abierta (N.A) y normalmente cerrada (N.C) como se puede visualizar en la tabla 1.

Tabla1. Equivalencias entre funciones lógicas y válvulas neumáticas 3/2 N.A y N.C

\begin{tabular}{|c|c|}
\hline $\begin{array}{c}\text { Función y Símbolo } \\
\text { lógico }\end{array}$ & Vályula/conexión \\
\hline
\end{tabular}

Elaborado por: Grupo de Investigación

En cambio, las funciones AND y OR, se pueden obtener con la combinación de válvulas neumáticas de vías en conexiones serie y paralelo, además esta función también tiene sus equivalentes en válvulas neumáticas especiales denominadas de simultaneidad y selectoras como las descritas en la tabla 2. 
Tabla 2. Equivalencias entre funciones lógicas y válvulas neumáticas de simultaneidad y selectora

\begin{tabular}{|c|c|c|}
\hline $\begin{array}{c}\text { Función y Símbolo } \\
\text { lógico }\end{array}$ & Conexión & $\begin{array}{c}\text { Equivalencia } \\
\text { neumática }\end{array}$ \\
\hline AND & & \\
\hline
\end{tabular}

Elaborado por: Grupo de Investigación

En el lenguaje de los circuitos neumáticos las "señales blocantes" son aquellas señales que actúan simultáneamente en una válvula de vías (distribuidores), con pilotaje neumático para generar la conmutación de sus posiciones. El bloqueo significa que la válvula no generara conmutación cuando tenga la presencia de presión tanto por $\mathrm{A}$, como por $\mathrm{B}$, quedándose en la posición que recibió la última señal, como se indica en la tabla 3.

Tabla 3. Válvula con pilotaje neumático tanto por A y B, con lo que genera el bloqueo.

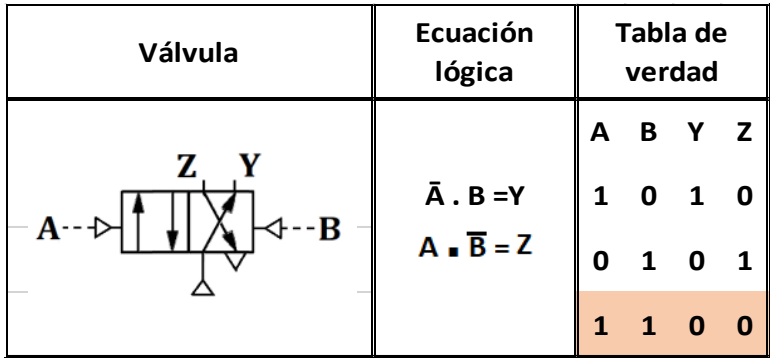

Elaborado por: Grupo de Investigación

Para la anulación de las señales blocantes pueden establecerse dos sistemas:

1) Anulación del efecto de la señal, y

2) La eliminación de la señal. 
Al analizar el primer sistema no se trata de abolir la señal blocante sino de dominarla por otra señal mayor, para ese cometido se dispone de las siguientes opciones: Válvula diferencial con accionamiento neumático, que tiene los dos pilotajes de distinto diámetro, de forma que con una misma presión aplicada a ambos la fuerza $(\mathrm{F}=\mathrm{P} \times \mathrm{S})$ es mayor en el pilotaje de mayor diámetro $(\mathrm{A}+)$, Si la señal blocante se aplica al pilotaje de menor diámetro(A-) al presentarse la señal en el otro pilotaje dominara a la señal blocante modificando la posición de la válvula. Como se puede observar en el literal a) de la figura1.

Otra forma de anular los efectos de la señal blocante es con un reductor de presión. En figura 1 ítem b) se presenta la conexión que se debe aplicar a la válvula bi-estable principal o de potencia con pilotaje neumático.

Con respecto al segundo sistema, para eliminar la señal blocante, puede utilizarse:

Válvulas de accionamiento mecánico unidireccional, conocida también como válvula de rodillo abatible, como la del literal c) en la figura 1.

Temporizadores neumáticos de impulsos normalmente abiertos como el representado en la figura 1 literal d).

Válvulas con accionamiento neumático del tipo bi-estable (memoria), cuyo símbolo se indica en la figura 1 literal e). Es un método muy utilizado por su seguridad, puesto que la señal sólo está presente en el momento en que se necesita. Con las señales se pueden realizar además muchas combinaciones. Así, por ejemplo, pueden anularse varias señales al mismo tiempo con el consiguiente ahorro de componentes de mando.

Figura 1. a) Válvula diferencial, b) conexión del reductor de presión, c) válvula de rodillo abatible, d) temporizador de impulsos y e) válvula bi-estable con pilotaje neumático

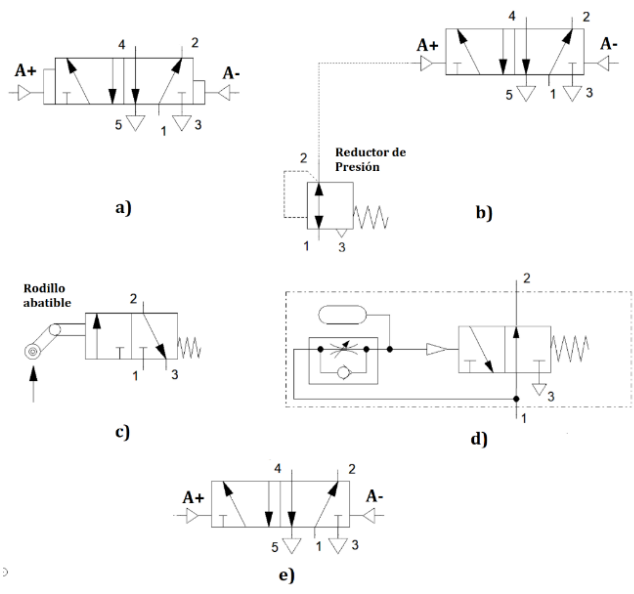

Elaborado por: Grupo de Investigación 
En el método que se propone en este trabajo, se utilizan válvulas auxiliares $\left(\mathrm{V}_{\mathrm{A}}\right)$, que eliminaran el número de señales blocantes $(\mathrm{N})$, estas son generalmente válvulas 4/2 o 5/2 bi estables de pilotaje neumático, las mismas que se pueden determinar de acuerdo a la siguiente expresión:

$\mathrm{V}_{\mathrm{A}}=\mathrm{N}-1$

\section{Importancia del problema.}

Enel campo del diseño neumática utilizamos diferentes procesos en los cuales las señales blocantes están presentes es por ello que se necesita que dichas señales sean superadas de una manera que optimice el proceso tanto neumáticamente como económica.

El proceso de optimización por medio de un método que nos permita la utilización del menor de los recursos disponibles no solo lo haría que se reduzca econicamente el proyecto sino que resulta un ahorro y mejor distribución de la presión y caudal del fluido disponible lo que nos permite obtener fuentes generadores de menor potencia.

La importancia de tener métodos que solucione este tipo de señales blocantes en el proceso de diseño circuital radica en reemplazar a los sistemas antiguos por técnicas más eficientes.

\section{Metodología.}

Consideraciones para la elaboración del diagrama K-V

Para usar la técnica del diagrama K-V para el diseño de un circuito neumático, se toma el diagrama de movimiento trayectoria - paso, el mismo que se obtiene de la secuencia de trabajo que describe el funcionamiento del automatismo, para fines de aplicación del Diagrama K-V se planteara el requerimiento formulado en la figura 2.

Figura 2. Automatismo para elevar cajas con bandas transportadoras y cilindros neumáticos de doble efecto.

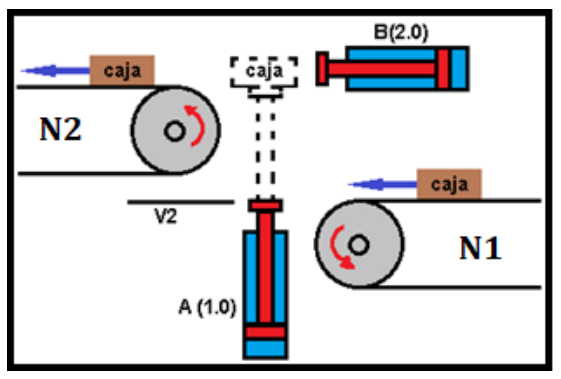

Elaborado por: Grupo de Investigación. 
El sistema está compuesto por dos cintas transportadoras y un elevador neumático automático. El elevador neumático eleva paquetes desde la cinta transportadora $\mathrm{N}^{\circ} 1$ hasta la cinta transportadora $\mathrm{N}^{\circ} 2$.

Cuando un paquete llega a la superficie superior del cilindro A (1.0) activa al sensor V2, el que opera el cilindro A, el cual levanta el paquete. El cilindro B (2.0) entonces reacciona, empujando el paquete a la cinta transportadora $\mathrm{N}^{\circ}$ 2, para retornar el cilindro A, primero debe regresar el cilindro $\mathrm{B}$.

En este caso, los dos cilindros A (1.0) y B (2.0) se van a controlar en forma secuencial para elevar una caja desde una banda transportadora a otra banda transportadora, de la cual se describe la secuencia A+/ B+/ B-/ A-.

Debajo de la línea correspondiente a cada paso (en la figura 3) se les ha dado una designación al estado requerido de la válvula de impulsos (5/2 de doble accionamiento neumático) y de las válvulas de disparo (3/2 de accionamiento mecánico) para regular los cilindros hacia la dirección deseada de su movimiento.

Figura 3. Diagrama de movimientos trayectoria pasos para la secuencia $A+/ B+/ B-$ /A+.

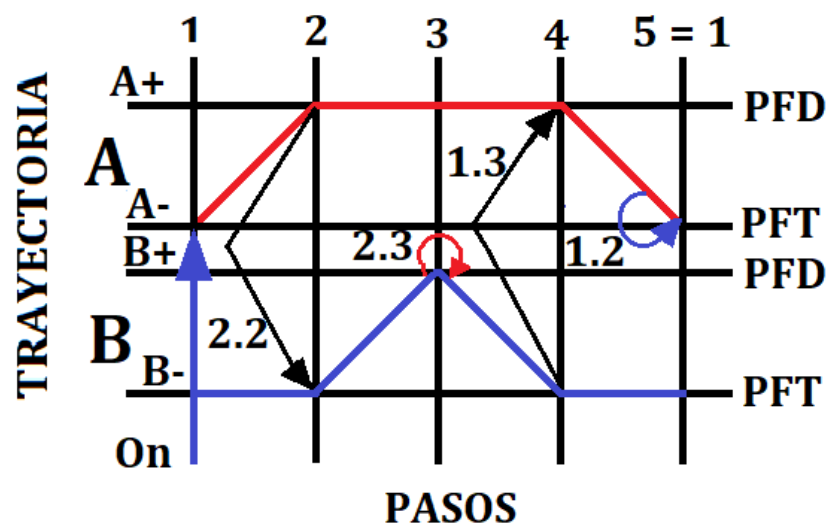

Elaborado por: Grupo de Investigación.

En la siguiente figura 4 se ilustran los elementos neumáticos básicos para los cilindros A y $\mathrm{B}$, junto con sus detectores de posiciones extremas. La válvula 1.1 comanda al cilindro A y la válvula 2.1 comanda al cilindro B. Las salidas de las válvulas 1.1 y 2.1 hacia los 
cilindros son las lumbreras 2 y 4 de cada una. Dichas salidas tienen dos valores posibles de presión: compresor (1) y retorno a la atmósfera (0) para cada una de las lumbreras y se mantienen en esos valores hasta nueva orden, es decir, 1.1 y 2.1 son elementos con memoria (válvula 5/2).

Figura 4. Elementos básicos para el automatismo A+/B+/B-/A-.

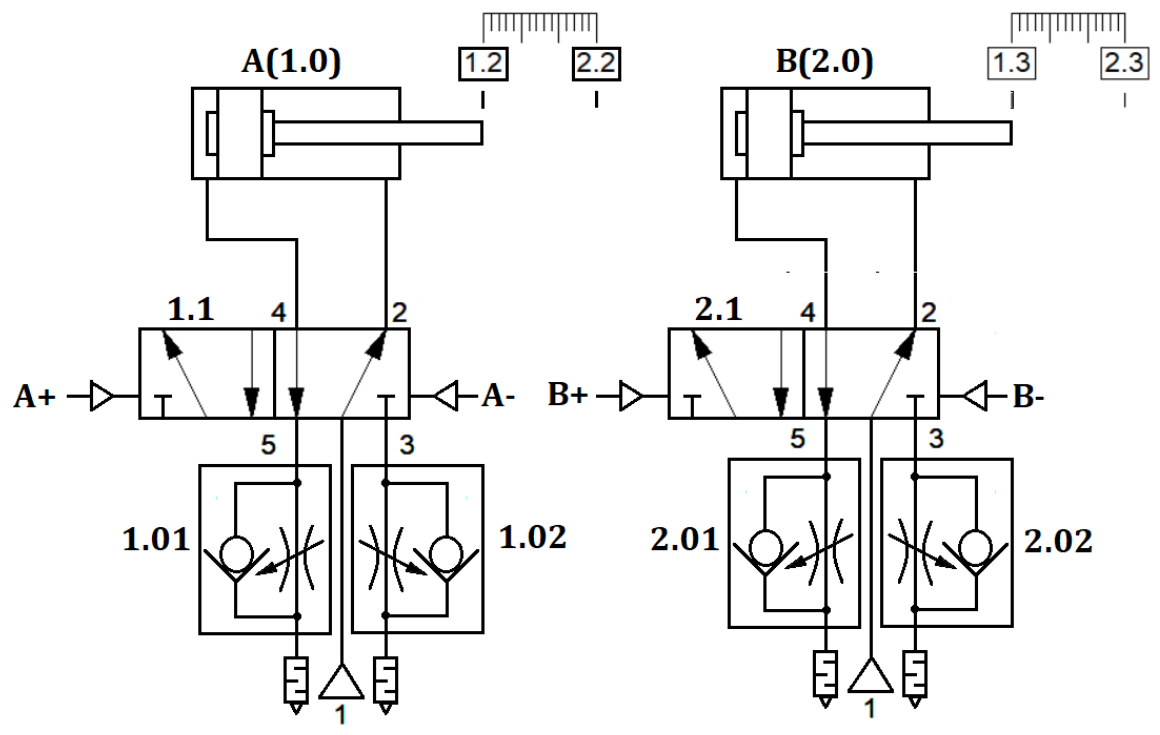

Elaborado por: Grupo de Investigación.

Para la solución del transporte de las cajas en las bandas se definen:

Entradas:

On: mando de inicio pulsador $3 / 2$.

1.2: detector en la posición final trasera (PFT), del cilindro A, que da señal para que el cilindro A avance a su posición final delantera (PFD).

1.3: detector en la PFT del cilindro $B$, que da señal para que el cilindro A retorne a su PFT 2.2: detector en la PFD del cilindro $A$, que da señal para que el cilindro $B$ avance a su PFD. 2.3: detector en la PFT del cilindro B, que da señal para que el cilindro B retorne a su PFT

Salidas:

A+: pilotaje de la válvula 1.1 para que el cilindro A avance de la PFT a la PFD, en donde la lumbrera 4 tendrá presión (1) y la lumbrera 2 retorno al ambiente (0). 
A-: pilotaje de la válvula 1.1 para que el cilindro A retorne de la PFD a la PFT, en donde la lumbrera 4 tendrá retorno al ambiente (0) y la lumbrera 2 presión (1).

B+: pilotaje de la válvula 2.1 para que el cilindro B avance. de la PFT a la PFD, en donde la lumbrera 4 tendrá presión (1) y la lumbrera 2 retorno al ambiente (0).

B-: pilotaje de la válvula 1.1 para que el cilindro A retorne de la PFD a la PFT, en donde la lumbrera 4 tendrá retorno al ambiente (0) y la lumbrera 2 presión (1).

A partir del diagrama trayectoria-pasos de la figura 3 se determina el funcionamiento del sistema de transporte da cajas por medio de bandas:

En el paso 1 se encuentran los estados iniciales, donde los cilindros se encuentran en el estado de reposo o en las PFT.

Del paso 1 al paso 2 el cilindro A pasa de la PFT a la PFD por la señal de 1,2 a la válvula $1.1(\mathrm{~A}+)$.

Del paso 2 al 3 el cilindro B pasa de la PFT a la PFD por la señal de 2.2 a la válvula 2.1 ( $\mathrm{B}+)$.

Del paso 3 al 4 el cilindro B pasa de la PFD a la PFD por la señal 2.3 a la válvula 2,1 (B-). Diseño de un circuito neumático con el diagrama K-V.

Para iniciar con el diseño del circuito neumático, se traza el diagrama $\mathrm{K}-\mathrm{V}$, en el cual las líneas horizontales indican el estado o condición de las señales (válvulas 3/2) y las columnas representan las salidas de la válvula auxiliar.

El número de columnas se determina por la trayectoria de flujo de la señal, en tanto que el número de cilindros determina el número de líneas horizontales. Por tanto, el número de líneas horizontales es igual a $2^{\mathrm{n}}$, en donde $\mathrm{n}=$ número de cilindros. Para dos cilindros, se trazan cuatro líneas horizontales en el diagrama $\mathrm{K}-\mathrm{V}$, ya que $2^{2}=4$ (es decir, $2^{\mathrm{n}}$ - número de campos).

La figura 5 representa el diagrama $\mathrm{K}-\mathrm{V}$ en donde se traza el diagrama de flujo de la señal con la dirección del flujo, al transferir todas las combinaciones de las señales (válvulas 3/2) a cada cuadrado de ese diagrama. A cada cuadrado en el diagrama $\mathrm{K}-\mathrm{V}$ se le da el nombre de campo. Cada campo cuadrado representa el estado de la válvula auxiliar (es decir, la válvula 4/2) A +, A, B+ y B-. 
En un campo sólo debe colocarse un estado y si sucede que en algún campo caen más de un estado (como $\mathrm{B}+\mathrm{y} \mathrm{A}$ - en la figura 5, entonces la nueva designación de la memoria se transfiere hacia una nueva columna, la cual representa la válvula auxiliar, se necesita ésta para efectuar la permutación del cilindro de la PFT a la PFD o vicevesa.

Figura 5: Diagrama K-V para la secuencia A+/B+/B-/A-.

\begin{tabular}{|l|l|l|}
\hline \multirow{2}{*}{1,3} & 1,2 & A+ \\
\cline { 2 - 3 } & 2,2 & B+ Ó A- O \\
\hline \multirow{2}{*}{2,3} & 2,2 & B- ’́ \\
\cline { 2 - 3 } & 1,2 & \\
\hline
\end{tabular}

Elaborado por: Grupo de Investigación.

Esta es la válvula que se transforma en una selectora de grupo: una válvula bi estable de pilotaje neumático para cortar la línea de energía hacia el conjunto de válvulas que dan las señales en un grupo y energizar otro grupo que necesita ser cambiado, como en este caso, en el cual se necesita cambiar las señales de B+ hacia B- y de A- hacia A+.

Ésta válvula se designa como $V_{A}$, con sus dos estados $V_{A}$ y $V_{A}$, en donde se usan $V_{A}+y$

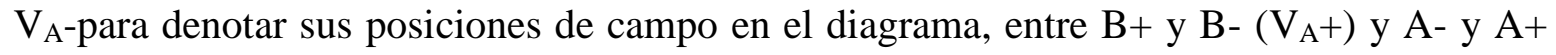
$\left(\mathrm{V}_{\mathrm{A}^{-}}\right)$. Las válvulas encargadas de emitir las señales se designan como 1.2, 1.3, 2.2, 2.3 , como ya se ha mencionado en las entradas de la figura 4.

El estado del cilindro se representa por medio de $\mathrm{A}+, \mathrm{A}-, \mathrm{B}+, \mathrm{B}-$, en donde "+" representa el avance del cilindro (de PFT a PFD) y “-” representa el retorno de ese cilindro (de PFD a PFT). En la figura 6 se presenta el nuevo diagrama K-V con la inclusión de la válvula auxiliar VA, la misma que prácticamente eliminara el control doble, que se produce en el diagrama de la figura 5, como también las ecuaciones lógicas que se generan.

Figura 6. a) Diagrama K-V con la dirección de flujo y b) ecuaciones lógicas que se generan.

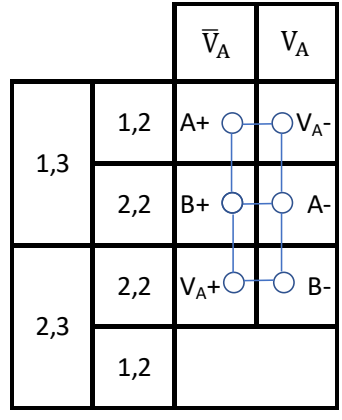

\begin{tabular}{|l|}
\hline Ecuaciones lógicas \\
\hline$A+$ \\
\hline$B+$ \\
\hline$V_{A}+$ \\
\hline$V_{A}-$ \\
\hline$A-$ \\
\hline$B-$ \\
\hline
\end{tabular}

Elaborado por: Grupo de Investigación. 
En la figura 7 se observa la disposición de la válvula auxiliar $\left(\mathrm{V}_{\mathrm{A}}\right)$, y aplicando el teorema De Morgan se simplifican las ecuaciones generadas en el diagrama $\mathrm{K}-\mathrm{V}$, quedando establecido de la siguiente manera las ecuaciones lógicas finales simplificadas:

Figura7 Vávula de auxiliar por el método de Morgan.

$$
\begin{gathered}
A-=\overline{V_{A}}, \text { y } A+=1,3 \cdot V_{A} \\
B+=1,3 \cdot V_{A} \\
B-=1,3 \cdot V_{A}
\end{gathered}
$$

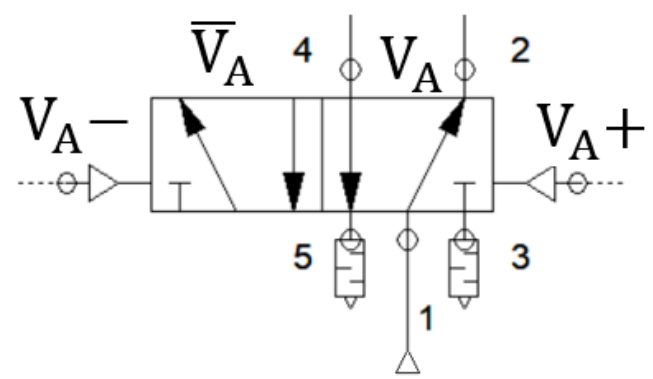

Elaborado por: Grupo de Investigación.

\section{Resultados.}

Como se puede ver en la figura número 3 nos muestra el diagrama espacio - fase nos indica que tenemos en el sistema una señal bloqueante la cual podemos ver solucionada a través del método Karnaugh - Veitch (K-V), el cual a diferencia de otros métodos como lo son: la técnica de mando abatible, la técnica de temporización, memoria auxiliar o cascada las cuales no son aplicables a todos los sistemas, por lo tanto tenemos que el método K-V no brinda mandos secuenciales de alta confiabilidad.

En la figura 8 podemos observar en el diagrama espacio-fase que la señal bloqueante es resuelta a través del método observando a su vez que se convierte en un proceso totalmente automático.

Considerablemente el número de válvulas utilizada en este método reduce de manera circunstancial en relación a los ya mencionados. La posición de conmutado en dicho diagrama nos permite observar que en la fase 2 y 8 está solucionada como en la fase 4 , con lo cual las señales blocantes son solucionadas de una manera eficiente. 
Figura 8. Diagrama espacio-fase por medio del método K-V.

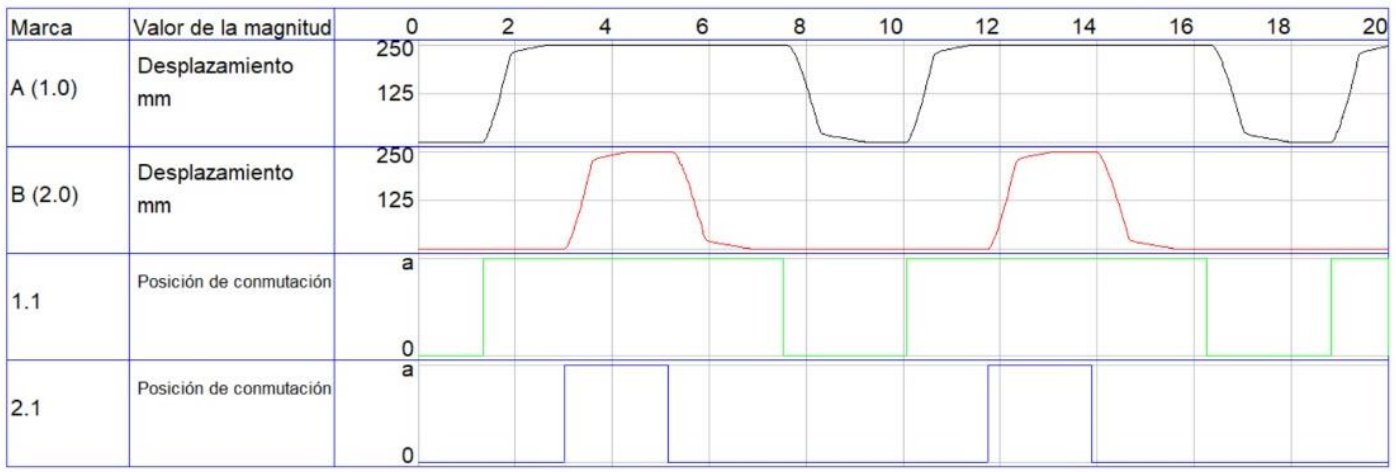

Elaborado por: Grupo de Investigación

A continuación se procede a observar como el método estructura el proceso antes descrito, en la figura 9 tenemos el circuito en estado de reposo estando los cilindros A y B en reposo antes del inicio del circuito neumático.

Figura 9. Circuito en estado de reposo.

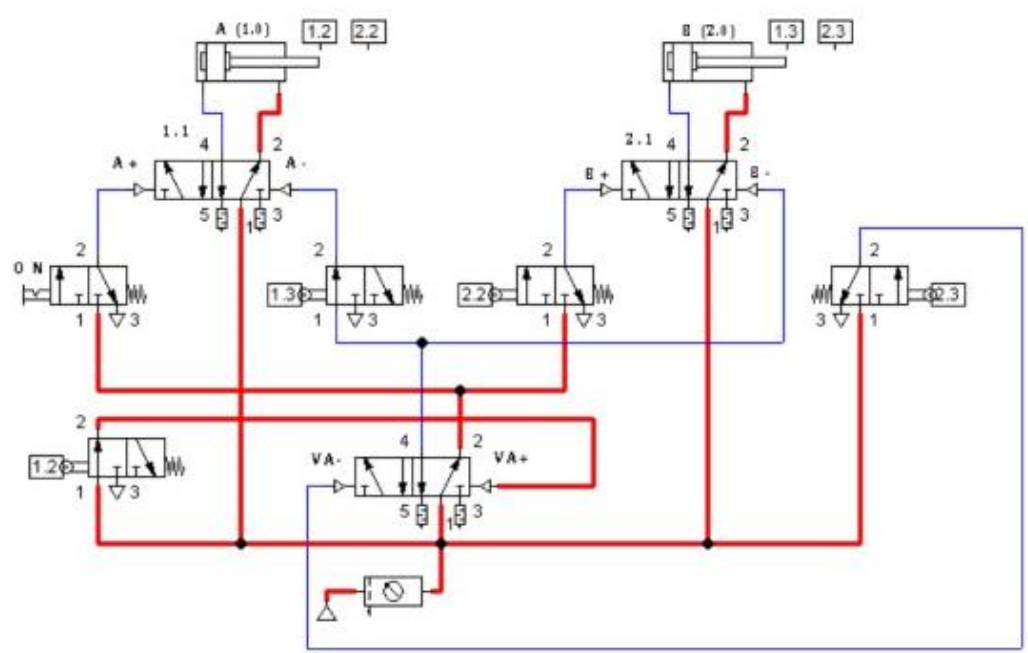

Elaborado por: Grupo de Investigación.

En la figura 10 podemos observar que al enclavar la válvula $3 \times 2$ que en este caso es ON (incio de secuencia), el flujo de aire permite al cilindro A iniciar su posicionamiento en al final de carrera 2.2 
Figura 10. Inicio de la secuencia de activación "on" y vástago del cilindro "A" en la posición final delantera.

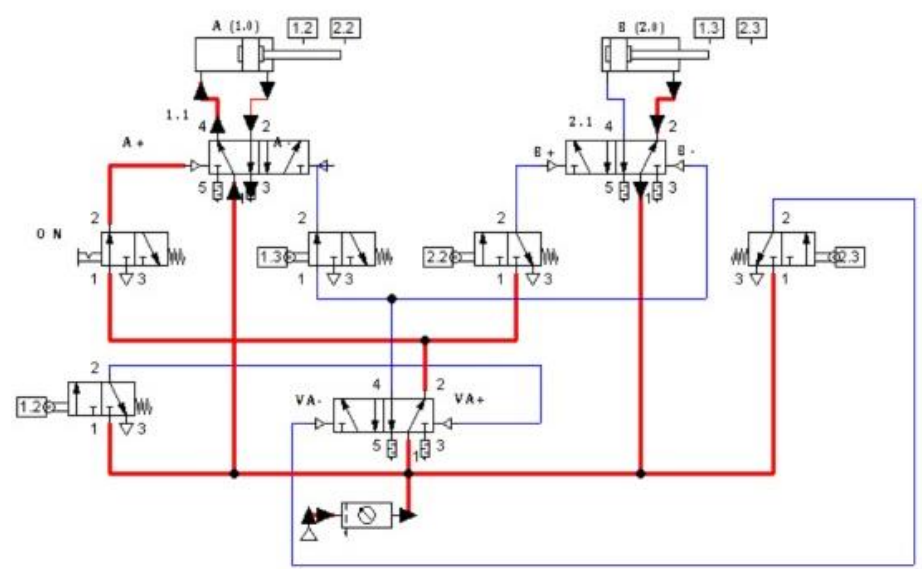

Elaborado por: Grupo de Investigación.

Como obsevamos en la figura 11 después de la activación del final de carrera 2.2 el fluido se distribuye en la válvula que activa la salida del cilindro B hasta su respectivo final de carrera 2.3.

Figura 11. Activación de 2.2 y avance del cilindro B.

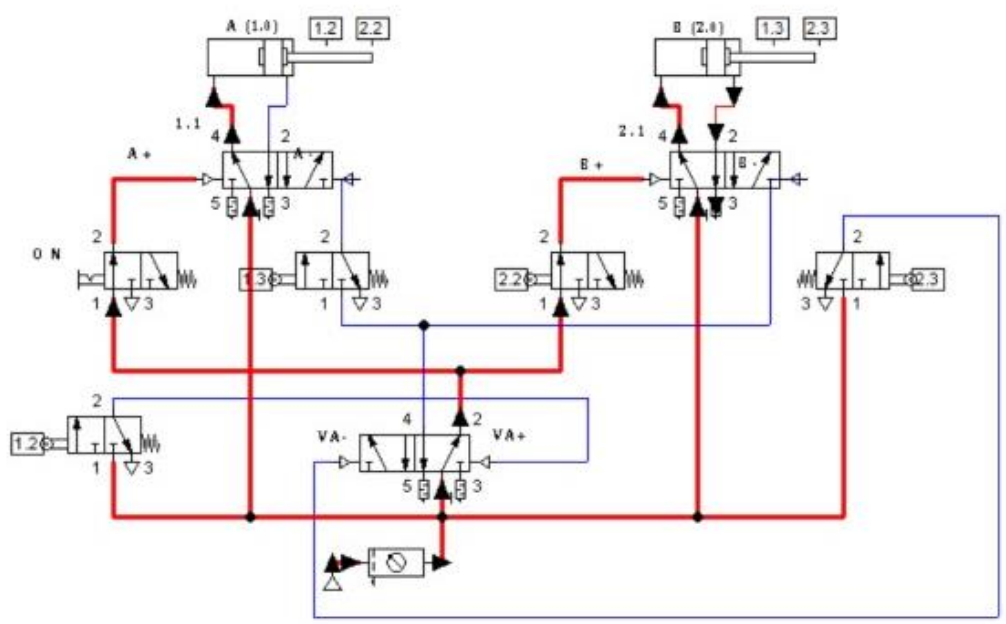

Elaborado por: Grupo de Investigación.

En la figura 12 podemos ver que al activar el final de carrera 2.3 nos permite activar la válvula auxiliar y nos permite el retornode la valvula $\mathrm{B}$ en la misma señal con lo que eliminamos la señal blocante aquí provista con la particularidad que obtenemos después el 
retorno del cilindro $\mathrm{B}$ que el ciclo antes establecido se vuelva a repetir haciéndose un bucle automatico.

Figura12. Retorno del cilindro A.

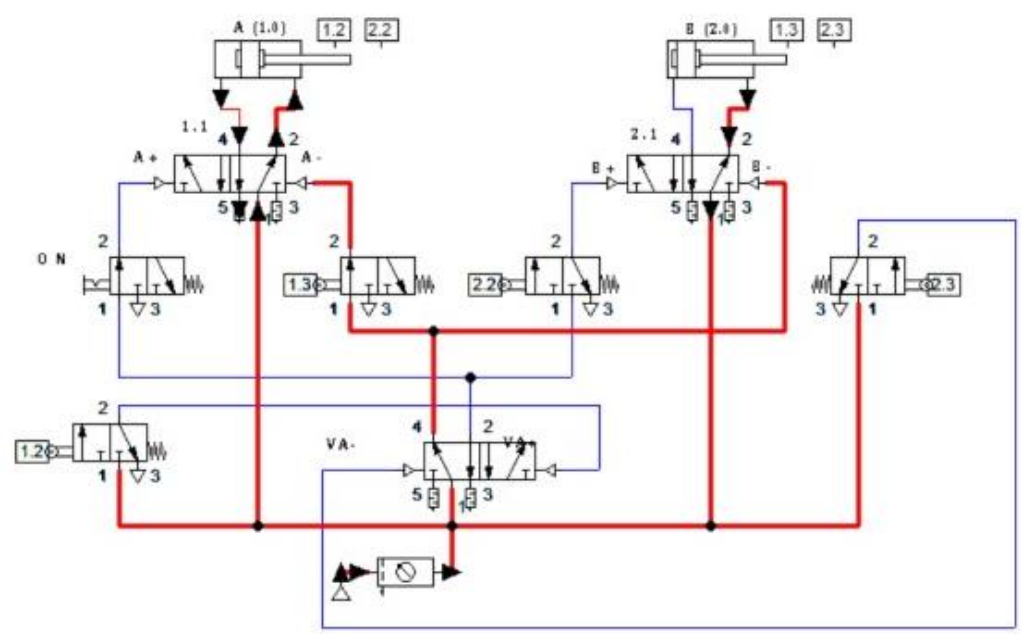

Elaborado por: Grupo de Investigación.

\section{Discusión.}

Los diagramas K-V que se reducen a la simplificación de los problemas como se puede ver en el caso propuesto se lo puede solucionar al aplicar este sistema a través de la división de la secuencias en dos partes diferentes como se lo puede observar en la figura 5.

Al nosotros tener una secuencia la secuencia $A+/ B+/ B-/ A-$, donde procemos a separar las señales blocantes y es ahí donde aplicamos el diagama $\mathrm{K}-\mathrm{V}$ lo que nos permite obtener las siguientes ecuaciones lo que nos ayuda a terminar este proceso de la eliminación de las señales bloqueantes. Cabe recalcar que este tipo de solución es aplicable tanto a los grandes y pequeños esquemas.

Tabla No1 Ecuaciones Lógicas.

\begin{tabular}{l}
\hline Ecuaciones lógicas \\
\hline$A+$ \\
$B+$ \\
$V_{A}+$ \\
$V_{A}-$ \\
$A-$ \\
$B-$ \\
\hline
\end{tabular}

Elaborado por: Grupo de Investigación. 


\section{Conclusiones.}

- En la gran mayoría de estudios de neumática las válvulas bi-estables son ampliamente utilizadas las que no responden a una señal mientras tenga presente la contraria a lo que comúnmente se llama una señal bloqueante. Uno de los procesos en los cuales estas señales se solucionan son temporizandolas, lo cual no es recomendado ya que este tipo de soluciones no son exactas al movimiento que deseamos y necesitamos otros tipos de componentes lo cual encarece el sistema, y en otros casos el número se vuelve numeroso aumentando su costo.

- Las señales bloqueantes a través del método K-V, es decir la lógica booleana, podemos simplicar el proceso de solución tradicionales es necesario aplicar esta metodología ya que nos oermite solucionar los pequeños y grandes sistemas neumáticos que se presentan a diario en el diseños de sistemas.

\section{Agradecimiento.}

Esta investigación no habría sido posible sin el apoyo de la empresa SICARSA por lo que agradecemos la disposición por buscar mejoras tecnológicas por el bien de la sociedad. Además este trabajo investigativo es el fruto del esfuerzo conjunto de conocimientos y experiencias laborales de los Ingenieros Elvis Arguello, José Pérez y Fabián Sánchez. Con estas líneas queremos mostrar nuestro agradecimiento a todas aquellas personas que supieron orientar nuestra investigación de una manera exitosa.

\section{Referencias bibliográficas.}

- Hesse, Stefan. Aire Comprimido Fuente de Energía by Festo AG y Co. (2002).

- Meixner, H., Introducción en la Neumática, Manual de Estudio. FESTO, 1988.

- Carnicer Royo, Enrique. Aire Comprimido. Ed. Paraninfo (1990), Madrid.

- Serrano, Nicolás. Neumática: 1a ed. España, Thompson, 2009.

- Dorf y Bishop, 2008. Modern Control Systems. 11th Edition, Prentice Hall, 2008.

- De las Heras, S., Instalaciones Neumáticas. Editorial UOC, 2003

- FESTO, Introducción a la Electroneumática. 1990.

- Hessen, S., 99 ejemplos prácticos de aplicaciones neumáticas. FESTO, 2000.

- Cembranos Nistral, Florencio Jesus. Automatismos eléctricos, neumáticos e hidráulicos. Madrid: Paraninfo. 
Para citar el artículo indexado.

Sánchez E., Arguello E., Pérez J. \& Noboa G., (2018). Aplicación de los diagramas de karnaugh - veitch, en el diseño de circuitos neumáticos con señales blocantes. Revista electrónica Ciencia Digital 2(2), 458-472. Recuperado desde: http://cienciadigital.org/revistacienciadigital2/index.php/CienciaDigital/article/view/113/10 $\underline{4}$

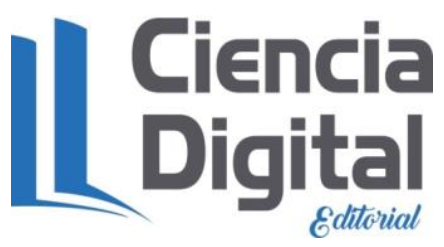

El artículo que se publica es de exclusiva responsabilidad de los autores y no necesariamente reflejan el pensamiento de la Revista Ciencia Digital.

El articulo queda en propiedad de la revista y, por tanto, su publicación parcial y/o total en otro medio tiene que ser autorizado por el director de la Revista Ciencia Digital.
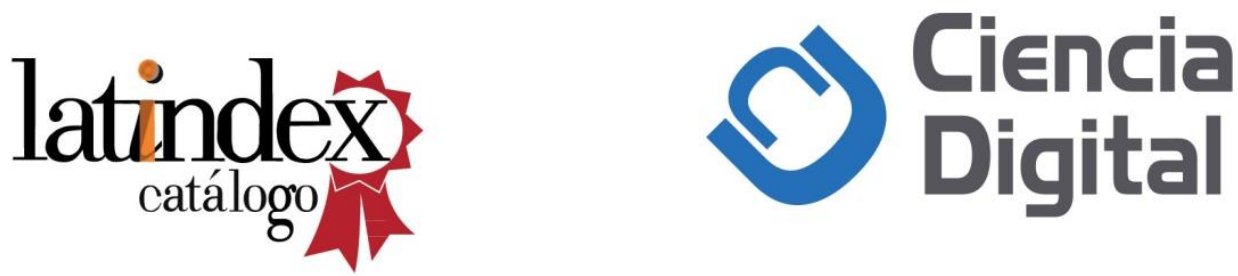